\title{
Viscous Drag Reduction with Surface-Embedded Grooves
}

\author{
Jovan Jovanović , Bettina Frohnapfel and Antonio Delgado
}

\begin{abstract}
Turbulent drag reduction in wall-bounded flows is investigated experimentally by considering the dynamic effects provoked by large variation of anisotropy in the velocity fluctuations. It is shown that high drag reduction is obtained when the velocity fluctuations near the wall reach the statistically axisymmetric state with invariance under rotation about the axis aligned with the mean flow. Deductions based on the analysis of near-wall turbulence leads to the design of the grooved surface topology, for which it is demonstrated experimentally that can produce a maximum drag reduction of $\mathrm{DR} \simeq 25 \%$. The drag reduction effect persisted in a narrow range of flow velocities and for the reported experimental conditions correspond to groove dimensions of about 0.8 viscous length-scale.
\end{abstract}

\section{Introduction}

A significant portion of the drag which counteracts the motion of a body through a fluid is generated in the thin viscous region close to the solid boundary where the flow is nearly always turbulent. The viscous contribution to the total drag amounts to about $50 \%$ on commercial aircraft, $90 \%$ on underwater vehicles and almost $100 \%$ for pipe and channel flows [1]. If we consider current trends in the crude oil prices, worldwide expansion of public transport and initiatives for reduced pollution of the

Jovan Jovanović

Institute of Fluid Mechanics, Friedrich-Alexander University Erlangen-Nuremberg, Germany,

e-mail: jovan.jovanovic@1stm.uni-erlangen.de

Bettina Frohnapfel

Center of Smart Interfaces, Darmstadt University of Technology, Petersenstraße 32,

64287 Dratmstadt, Gremany, e-mail: frohnapfel@csi.tu-darmstadt.de

Antonio Delgado

Institute of Fluid Mechanics, Friedrich-Alexander University Erlangen-Nuremberg, Germany,

e-mail: antonio.delgado@1stm.uni-erlangen.de 
environment, the beneficial outcomes of reducing viscous drag and therefore energy consumption are obvious. The fluid mechanics community is desperately searching for practical solutions in order to satisfy the expectations of society using all tools and techniques available, but practically no success has been achieved so far.

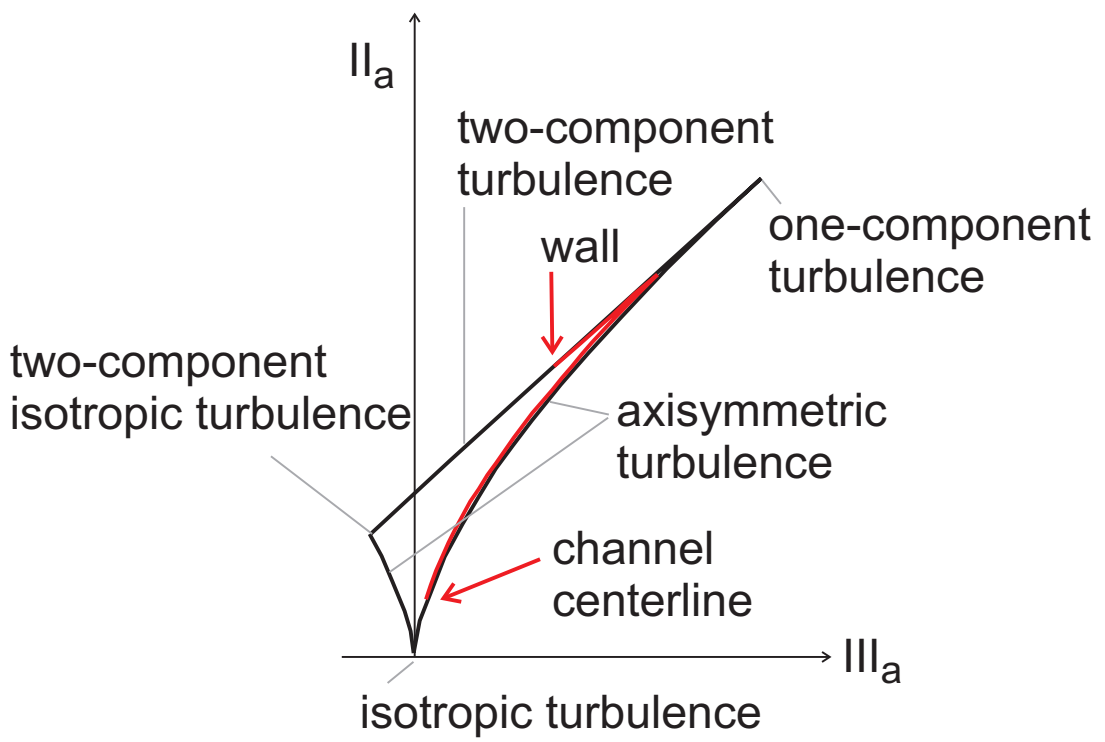

Fig. 1 Anisotropy-invariant map according to Lumley [2] with the trajectory of a turbulent channel flow (in red). The arrow "wall" marks the position of the turbulence state at the wall. A movement of this point towards the upper right-hand corner (one-component turbulence) along the twocomponent line marks increasing anisotropy of turbulence as it is commonly found for a variety of different drag reduced flows.

\section{The Mechanism of Turbulent Drag Reduction}

The origin of turbulence in wall-bounded flows can be analyzed by looking into the evolution of anisotropy in the turbulent stresses $\overline{u_{i} u_{j}}$, which can be quantified using the anisotropy tensor ([2]) defined as $a_{i j}=\overline{u_{i} u_{j}} / q^{2}-1 / 3 \delta_{i j}$ (where $q^{2}=\overline{u_{s} u_{s}}$ ) and its scalar invariants $\mathrm{II}_{\mathrm{a}}=a_{i j} a_{j i}$ and $\mathrm{III}_{\mathrm{a}}=a_{i j} a_{j k} a_{k i}$. A plot of $\mathrm{II}_{\mathrm{a}}$ versus $\mathrm{III}_{\mathrm{a}}$ for axisymmetric turbulence, $\mathrm{II}_{\mathrm{a}}=3 / 2\left(4 / 3\left|\mathrm{III}_{\mathrm{a}}\right|\right)^{2 / 3}$, and two-component turbulence, $\mathrm{II}_{\mathrm{a}}=2 / 9+2 \mathrm{III}_{\mathrm{a}}$, defines the anisotropy-invariant map shown in figure 1 which bounds all physically realizable turbulence ([2]). The two curves in this figure represent axisymmetric turbulence. The right-hand curve corresponds to turbulence with the streamwise intensity component larger than in the other two directions, $\overline{u_{1}^{2}}>\overline{u_{3}^{2}}=\overline{u_{2}^{2}}\left(\mathrm{III}_{\mathrm{a}}>0\right)$, and the left-hand curve corresponds to axisymmetric turbu- 
lence with $\overline{u_{1}^{2}}<\overline{u_{3}^{2}}=\overline{u_{2}^{2}}\left(\mathrm{III}_{\mathrm{a}}<0\right)$. Along the straight line resides two-component turbulence. The limiting states of turbulence are located at the corner points on the right- and left-hand sides of the anisotropy-invariant map and correspond to onecomponent turbulence and isotropic two-component turbulence, respectively.

In [3] and [4] it was shown that turbulent drag reduction is associated with an increase of the anisotropy of turbulence in the near-wall region for a number of different flow control techniques. In this region, where the continuity equation and a no-slip boundary condition at the wall dictate turbulence to be two-componental, the turbulence anisotropy is directly proportional to the ratio between streamwise $\left(\overline{u_{1}^{2}}\right)$ and spanwise $\left(\overline{u_{3}^{2}}\right)$ stress components: with the streamwise component generally larger than the spanwise one. The increase of turbulence anisotropy, which can be captured in the anisotropy-invariant map as shown in figure 1, is most naturally achieved with a suppression of the spanwise stress components which corresponds to forcing turbulence to satisfy axisymmetry simultaneously at large and small scales very close to the wall with invariance under rotation about the axis aligned with the mean flow [3].

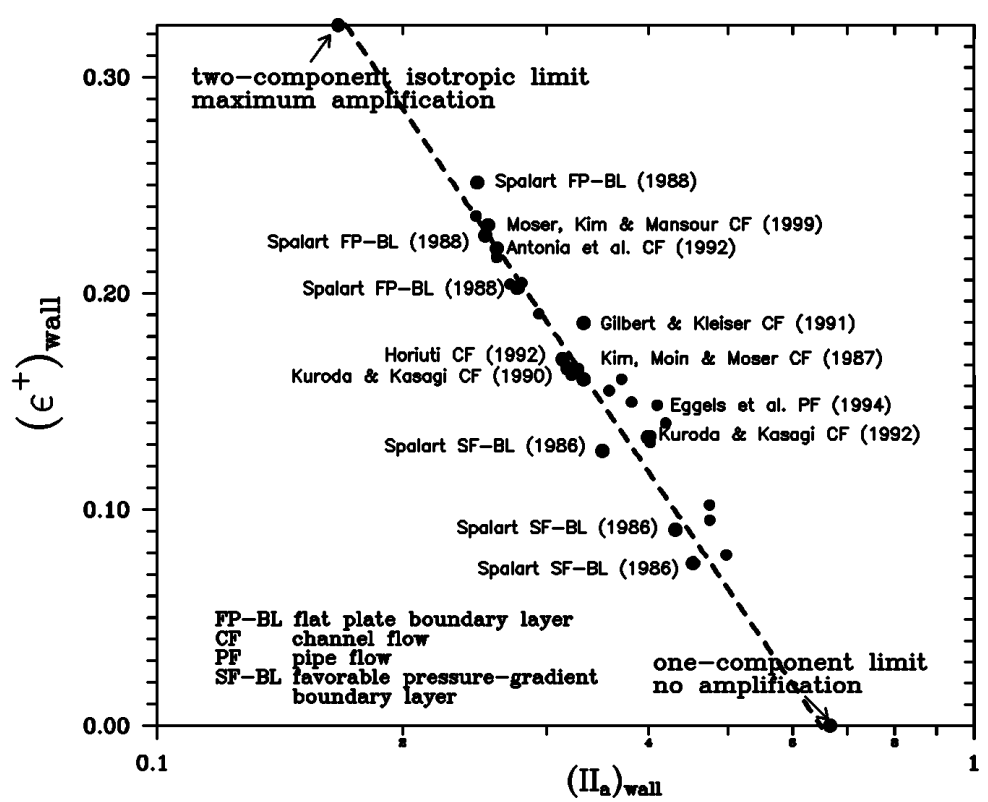

Fig. 2 Turbulent dissipation rate at the wall $(\varepsilon)_{\text {wall }}$ normalized with the wall shear velocity and the kinematic viscosity of the flow medium versus the anisotropy of turbulence at the wall $\left(\mathrm{II}_{\mathrm{a}}\right)_{\text {wall }}$. A best-fit line through the numerical data extrapolates fairly well the expected trend as the onecomponent limit $(\mathrm{II}=2 / 3)$ is approached [3]. 
For this special situation theoretical considerations and numerical simulations show that the dissipation rate must vanish at the wall, $\varepsilon_{\mathrm{wall}} \rightarrow 0$ ([3]). This fundamental deduction, which is displayed figure 2 , implies that as long as turbulence assume the one-component state at the wall the energy of turbulence $\left(k=1 / 2 q^{2}\right)$ cannot be amplified since $k$ grows as $k \rightarrow\left(\varepsilon_{\text {wall }} / v\right) x_{2}^{2} / 2$ as the wall is approached, $x_{2} \rightarrow 0$. Under such circumstances, the averaged total energy dissipation rate $\bar{\Phi}$ :

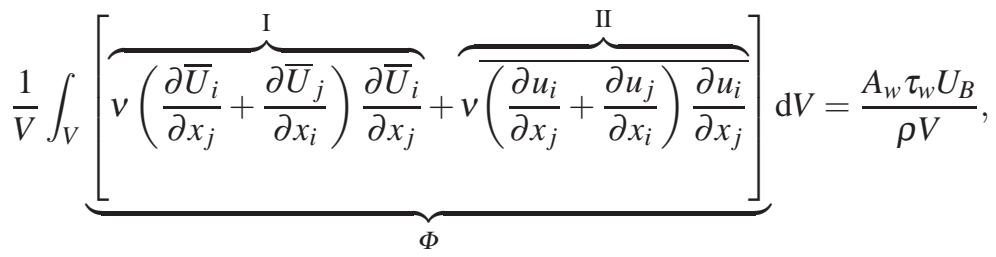

which is composed of the direct dissipation (I) and the turbulent dissipation $\varepsilon$ (II) and which can be evaluated from the work done against the wall shears stress $\tau_{w}$ per unit mass of the working fluid $\rho V$, where $A_{w}$ is the wetted surface area and $U_{B}$ is the bulk velocity, assumes a minimum at sufficiently high Reynolds numbers and a large drag reduction must occur as a logical consequence. This expectation results from an order of magnitude analysis, which shows that the largest contribution to $\bar{\Phi}$ is due to the turbulent dissipation, which reaches a maximum value at the wall.

\section{Experimental Investigations}

\subsection{Grooved Surface Topology}

A surface topology with grooves embedded in the wall and aligned in the streamwise direction as shown in figure 3 was designed. Inside a groove, the velocity fluctuations are suppressed not only in the normal but also in the spanwise direction due to the side walls and therefore it is expected that turbulence in the groove will tend toward the one-component limit at the wall. Direct numerical simulations confirm that such turbulence is almost axisymmetric with invariance to rotation about the axis aligned with the mean flow direction [5].

A grooved surface pattern was produced by milling grooves into flat metal plates and subsequent polishing of the plates. Groove dimensions of $h \approx 150 \mu \mathrm{m}$ with a separation of $2 h \approx 300 \mu \mathrm{m}$ were produced in metal plates of the dimensions $1.5 \mathrm{~m} \times$ $0.3 \mathrm{~m}$ which served as replacement for the smooth top and bottom walls of a twodimensional channel flow facility shown in figure 4. 


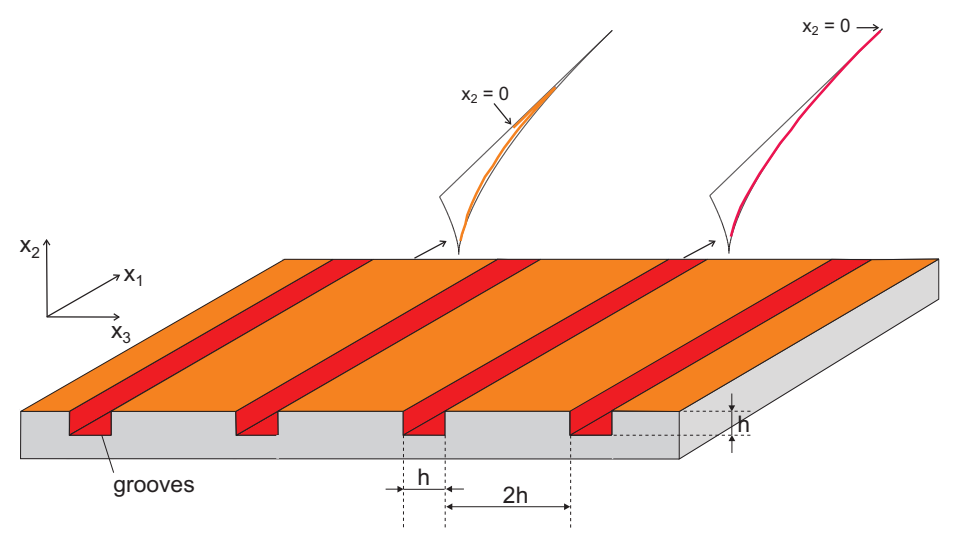

Fig. 3 Inside the grooves the flow is forced towards the one-component limit. Sketched are trajectories across the anisotropy-invariant maps for each portion of the surface.

\subsection{Channel Test Rig}

The channel flow facility is driven by a centrifugal blower. The air stream is guided through a well-designed settling chamber to ensure uniform flow condition at the channel inlet. On the suction side of the blower, a Venturi nozzle was located, which allowed the determination of the volume flow rate. The channel itself is $L=3.5 \mathrm{~m}$ long and $B=300 \mathrm{~mm}$ wide and can be adjusted to heights of $H=25,35$ and $41 \mathrm{~mm}$. At the channel inlet a trip is installed to ensure turbulent flow conditions and reproducible results at low Reynolds number. After the trip, a length of $0.7 \mathrm{~m}$ is provided for the flow development, which corresponds to $x_{1} / H=28.0,20.0$ and 17.0, respectively. The following $2.8 \mathrm{~m}$ of the channel comprise the test section which is equipped with 14 pressure taps of $300 \mu \mathrm{m}$ diameter at intervals of $200 \mathrm{~mm}$ located on both of the channel side walls. For the determination of the pressure drop over the channel length, the static pressure at the first measuring station (i.e. the one closest to the inlet of the channel) is compared with readings from subsequent measuring stations.

The test section is divided into two parts of equal length. In each part of the test section, the top and bottom walls are interchangeable so that smooth and grooved channel walls can be installed. To evaluate the drag reduction performance of the surface grooves, the pressure drop in a channel with and without grooves needs to be compared. To ensure identical operating conditions for both cases, an in line setup is chosen in which one part of the test section has smooth walls while the second part is equipped with grooved walls. Based on pressure drop measurements in the subsequent parts of the test section, drag reduction is evaluated according to:

$$
D R=1-\frac{(\Delta p / \Delta l)_{\text {grooved }}}{(\Delta p / \Delta l)_{\text {smooth }}} .
$$




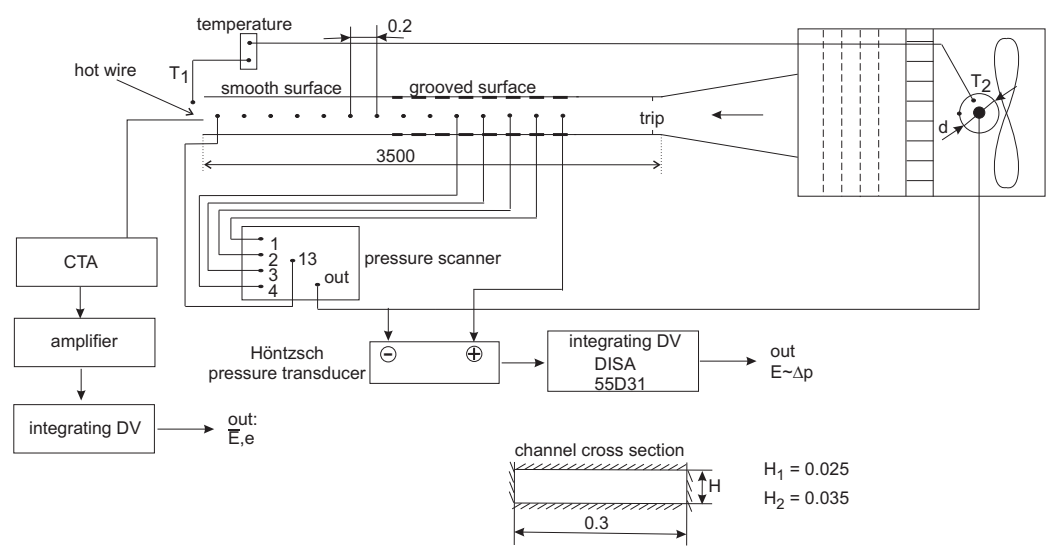

Fig. 4 Experimental set up and instrumentation used for drag reduction and turbulence measurements.

The grooved walls can either be inserted at the beginning or the end of the channel test section. In preliminary measurements it was established that the arrangement of grooved top and bottom walls followed by smooth ones yields the more conservative estimate of drag reduction. Therefore, measurements are carried out in this set-up. Further details on the measurement facility and measurement accuracy can be found in [6].

\subsection{Experimental Results}

The set of drag reduction measurements was carried out for the channel heights of $H=25,35$ and $41 \mathrm{~mm}$ with groove dimension of $h \approx 150 \mu \mathrm{m}$. Figure 5 shows all drag reduction data obtained. These measurements were carried out in a Reynolds number range of $R e_{b} \approx 1000-45000$. Over a wide range of Reynolds number the measured drag reduction deduced from pressure drop measurements is within the limit of the measurement accuracy. However, in very narrow range of flow velocities for three channel heights significant drag reduction of up to $25 \%$ are detected. The drag reduction data collapse if plotted versus the dimensionless groove size $h^{+}=$ $h u_{\tau} / v$ and show a common peak for a dimensionless groove size of $h^{+} \approx 0.8$. For grooves with $h \approx 150 \mu \mathrm{m}$ the corresponding Reynolds number where drag reduction occurs is in the range of $\operatorname{Re}_{b}=2300-3200$.

Ongoing experimental investigations of drag reduction by flow control of velocity fluctuations in the near-wall region of naturally developing boundary layers using surface embedded groves show that the drag reduction effect can be even increased at high Reynolds numbers [7]. These results support indirectly the mech- 


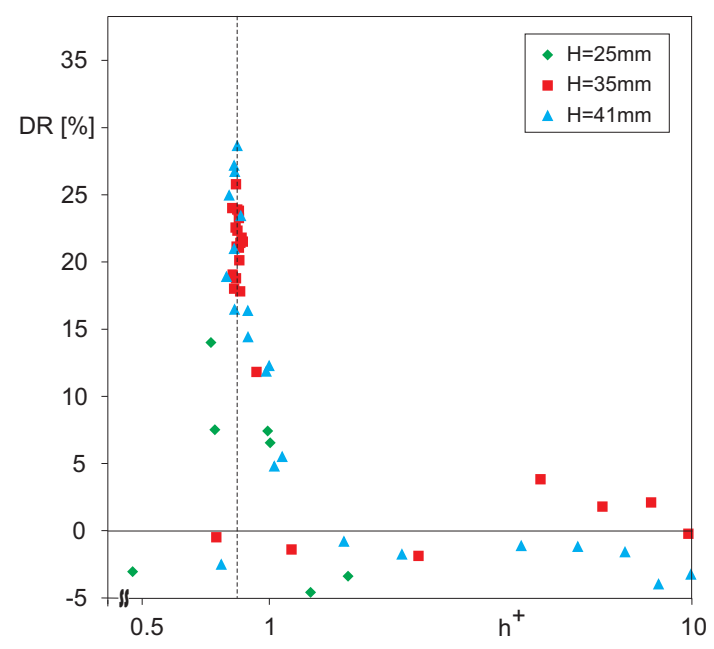

Fig. 5 Drag reduction versus dimensionless groove dimension for different heights of the channel test section(right).

anism responsible for laminar to turbulence transition and breakdown to turbulence elaborated by the authors [8], [9].

This work was sponsored by grant Jo 240/5-1 from the Deutsche Forschungsgemeinschaft. The authors gratefully acknowledge this support.

\section{References}

1. Lee, K.H., Cortelezzi, L., Kim, J., Speyer J.: Application of reduced-order controller to turbulent flows for drag reduction. Phys. Fluids 13, 1321, 2001.

2. Lumley, J.L.: Computational modelling of turbulent flows. Adv. Appl. Mech. 18, pp. 123-176, 1978.

3. Jovanović, J., Hillerbrand, R.: On the peculiar property of the velocity fluctuations in wallbounded flows. Thermal Science 9, pp. 3-12, 2005.

4. Frohnapfel, B., Lammers, P., Jovanović, J., Durst, F.: Interpretation of the mechanism associated with turbulent drag reduction in terms of anisotropy invariants. J. Fluid Mech. 577: 457-466, 2007.

5. Frohnapfel, B.: Flow control of near-wall turbulence. PhD thesis, University ErlangenNuremberg, Shaker Verlag, Aachen, 2007.

6. Frohnapfel, B., Jovanović, J., Delgado, A.: Experimental investigation of turbulent drag reduction by surface embedded grooves. J. Fluid Mech. 590: 107-116, 2007.

7. Jovanović, J., Frohnapfel, B. and Lienhart, H.: Further measurments of viscous drag reduction in a groove-modified channel flow. To be submitted to J. Fluid Mech., 2009.

8. Jovanović, J. and Pashtrapanska, M.: On the criterion for the determination transition onset and breakdown to turbulence in wall-bounded flows. J. Fluids Eng., 126, 626-633, 2004.

9. Jovanović, J., Frohnapfel, B., Škaljić, E. and Jovanović, M.: Persistence of the laminar regime in a flat plate boundary layer at very high Reynolds number. Thermal Science, 10, 63-96, 2006. 